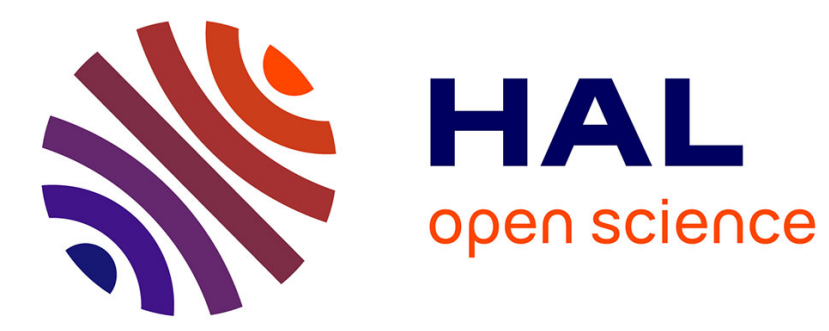

\title{
Bending waves in crumpled sheets
}

\author{
G. Seizilles, Elsa Bayart, Mohktar Adda-Bedia, A. Boudaoud
}

\section{To cite this version:}

G. Seizilles, Elsa Bayart, Mohktar Adda-Bedia, A. Boudaoud. Bending waves in crumpled sheets. Physical Review E: Statistical, Nonlinear, and Soft Matter Physics, 2011, 84 (6), 10.1103/PhysRevE.84.065602 . hal-03025810

\section{HAL Id: hal-03025810 https://hal.science/hal-03025810}

Submitted on 26 Nov 2020

HAL is a multi-disciplinary open access archive for the deposit and dissemination of scientific research documents, whether they are published or not. The documents may come from teaching and research institutions in France or abroad, or from public or private research centers.
L'archive ouverte pluridisciplinaire HAL, est destinée au dépôt et à la diffusion de documents scientifiques de niveau recherche, publiés ou non, émanant des établissements d'enseignement et de recherche français ou étrangers, des laboratoires publics ou privés. 


\title{
Bending waves in crumpled sheets
}

\author{
G. Seizilles, E. Bayart, M. Adda-Bedia, and A. Boudaoud \\ Laboratoire de Physique Statistique, Ecole Normale Supérieure, UPMC Paris 06, Université Paris Diderot, \\ CNRS, 24 rue Lhomond, F-75005 Paris, France
}

(Received 6 July 2011; published 27 December 2011)

\begin{abstract}
Crumpled paper has recently emerged as a model for disordered media. Here we use wave propagation to probe aluminum foils crumpled into balls made by hand or into cylinders obtained by confinement in a container. Surprisingly, the raw dispersion relations appear to differ from sample to sample. They correspond to bending waves that follow an effective path that is shorter than the distance between the input and output points. This can be interpreted in terms of two modes of propagation: slow bending waves and a fast mode whose possible origin is discussed. In addition, the effective paths behave differently in spheres and in cylinders. These results enable the characterization of the sample structure and point toward the geometric rigidity of the configurations.
\end{abstract}

DOI: 10.1103/PhysRevE.84.065602

PACS number(s): 46.70.De, 43.40.+s, 46.40.-f

Crumpled paper has become a playground for studies on disordered systems [1]. When strongly compressed, a thin sheet folds into a polyhedral-like shape, so that most of the energy is focused at the edges and vertices. Geometrical studies considered characterizations such as the roughness of unfolded sheets [2-4], fractal descriptors of crumpled balls [5-7], or the distributions of edge length [3,4,8,9]. Mechanical studies addressed the relation between the force applied on the crumpled ball and its volume [7,8,10,11]. Finally, acoustic studies analyzed the distribution of energy of acoustic pulses emitted when a sheet is crumpled [12-14]. Many important features were identified, such as the history-dependent behavior under mechanical loading, the wide distribution of energies, or the stacking of layers in crumpled configurations. However, the picture is far from being complete.

In other disordered media, wave propagation has been used as a tool to probe the system, featuring phenomena such as multiple paths or Anderson localization [15]. This motivated us to investigate whether the propagation of mechanical waves in a sheet yields information on its configuration. Thin sheets have two types of waves: in-plane nondispersive waves with a velocity comparable to the sound speed, and out-of-plane bending waves which are dispersive and much slower (for the same wave vector) than sound velocity. Consequently, in experiments at low forcing frequency, only bending waves are excited. Previous work on simple crumpled configurations-a tetrahedron [16] or a single vertex [17]—-showed that bending waves can be trapped in a facet or move around a vertex. Here we consider compact crumpled balls, and measure their dispersion relation. Our results may reveal features of wave paths that reflect the sheet configuration.

In order to avoid propagation through the container, we sought to obtain crumpled balls that remain folded with no confinement. We therefore worked with a ductile material, aluminum foils of thickness $h=20 \mu \mathrm{m}$ cut into squares with side length in the range $200-330 \mathrm{~mm}$. We note that ductility may make differences with the materials (paper or polyacetate) that were used in most previous studies on crumpling, in particular, aluminum foils display sharp and stiff folds. We generated isotropic crumpled balls by hand crushing foils into roughly spherical shapes (less than $20 \%$ in excentricity); the diameter of the ball $R$ was defined as the average of three diameters, and ranged from 35 to $85 \mathrm{~mm}$. We also sought a method for reproducible crumpling and were inspired by the experimental setup in Refs. [18] and [19]. The sheet is pulled through a hole of diameter $50 \mathrm{~mm}$, giving it a roughly conical shape, then it is put into a cylindrical container (radius 15 or $26 \mathrm{~mm}$ ) and compressed by a piston to a desired height $L$ in the range $20-50 \mathrm{~mm}$ (Fig. 1), yielding a crumpled cylindrical sample of centimetric size.

After making the sample, we glue two accelerometers on the centers of the crumpled cylinder faces (Fig. 1) or on two diametrically opposed points of the crumpled sphere. The accelerometers [Brüel and Kjær (B\&K) DeltraTron 4508 $\mathrm{B}$ 002] remain linear, within $5 \%$, in the frequency range 50-800 $\mathrm{Hz}$ that we investigated. The noise-to-signal ratio was in the range $1 \%-5 \%$. Waves are generated by an electromagnetic shaker (B\&K, Vibration Exciter 4809) bolted to the lower accelerometer. Thus the forcing area corresponds to the upper face of the accelerometer, a square of side length $10 \mathrm{~mm}$. The experiment is computer controlled with a LABVIEW interface. The input signal is sampled at $25 \mathrm{kHz}$ and amplified (B\&K Power Amplifier 2718). The input signal is a sinusoid of frequency $f$ with a Gaussian envelope of time $t$ proportional to $\sin (2 \pi f t) \exp \left[-\left(t-t_{0}\right)^{2} f^{2}\right]$ [Fig. 2(a)]. The choice of the envelope width was an optimum between having enough peaks in the signal and being able to discriminate the fastest propagation path (see below). Finally we ensured to remain in a linear regime.

First, we consider the flight time $\Delta t$ corresponding to the propagation between the two accelerometers. The comparison between the input and output signals [Fig. 2(a)] shows that the shape of the wave packet is roughly conserved up to the third or the fourth extremum, while showing significant damping. The additional peaks and the deformed envelope in the latest part of the output might be ascribed to multiple propagation paths, so that the use of correlation functions to estimate the $\Delta t$ turned out to be inaccurate. Therefore we measured the delay of the first extremum between the input and output, as shown in Fig. 2(a). We first checked the reproducibility of the results by plotting $\Delta t$ as a function of the forcing frequency $f$ for two identical samples made independently in the same experimental conditions [Fig. 2(a)] and found the agreement to be surprisingly good. Examples of $\Delta t(f)$ curves are shown in Fig. 2(b) for three different samples: two cylindrical and one spherical. In general, $\Delta t(f)$ increases up to a frequency of 

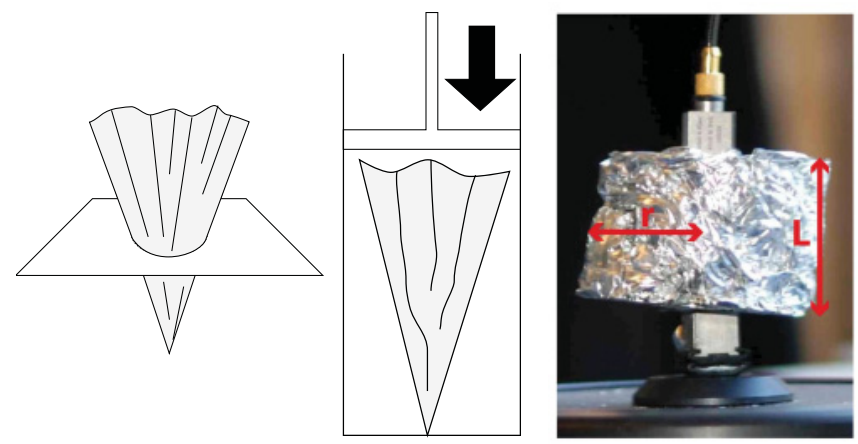

FIG. 1. (Color online) The experimental setup. Left-hand side: Setup for reproducible crumpling into a cylindrical sample; the aluminum foil is passed through a hole and then crushed by a piston into a cylindrical container. Right-hand side: Two accelerometers are glued at the center of the faces of the sample and the whole system is placed on a vibration exciter bolted to the lower accelerometer.

the order of $200 \mathrm{~Hz}$ and then decreases. Finally, $\Delta t$ increases with the size of the sample.

We now turn to the dispersion relation of the waves. The flight time $\Delta t$ directly yields the phase velocity $v_{\phi}=L / \Delta t$, $L$ being the distance between the two sensors. We first note that $v_{\phi}$ is in the range $10-100 \mathrm{~mm} / \mathrm{s}$ and is much smaller than the sound velocity in air or in aluminum; as a consequence, it is likely that we excite bending waves. The role of air can be evaluated using the ratio $\mu$ of mass of air entrained by vibrations and the mass of sheet. It is given by $\mu=\left(\rho_{a} / k\right) /\left(\rho_{s} h\right)$ [20], where $\rho_{a}$ and $\rho_{s}$ are, respectively, the densities of air and of the sheet, and $k=2 \pi f / v_{\phi}$ is the wave number which typically ranges from 10 to $160 \mathrm{~m}^{-1}$, so that most wavelengths are larger than the distance between the two sensors. Thus $\mu<1$ for $k>20 \mathrm{~m}^{-1}$, therefore entrained air is negligible for large enough wave numbers. Examples of dispersion relations are shown in Fig. 3(a), where the angular frequency $\omega=2 \pi f$ is plotted against $k$; when $k$ is large enough, they are consistent with the quadratic dispersion of the form $\omega=\alpha k^{2}$, characteristic of free bending waves. Indeed, if the (longitudinal) sound velocity in the material is $c$, its Poisson ration is $v$, and the thickness of the sheet is $h$, then the dispersion relation of bending waves takes the form

$$
\omega=c^{*} h k^{2} \quad \text { where } \quad c^{*}=\sqrt{\frac{1-2 v}{12(1-v)^{2}}} c .
$$

In the case of aluminum, $c=6 \times 10^{3} \mathrm{~m} / \mathrm{s}$ and $v=0.33$, so that the prefactor $\alpha$ of the dispersion relation should be $c^{*} h=0.03 \mathrm{~m}^{2} / \mathrm{s}$. The fits to the data give values of $\alpha$ between 0.1 and $0.6 \mathrm{~m}^{2} / \mathrm{s}$. Not only are the experimental prefactors approximately an order of magnitude too large, but also they vary with the dimensions of the sample. This suggests that the path of the waves differs from the straight line between the two sensors.

Consequently, we decided to extract the length of the path $l^{*}$ from the dispersion relation. The wave number must be redefined as $k=\omega \Delta t / l^{*}$. Using the dispersion relation (1), we obtain the effective length as $l^{*}=\Delta t \sqrt{c^{*} h \omega}$. Typical results are shown in Fig. 3(b). $l^{*}$ increases for $f<200 \mathrm{~Hz}$ and is roughly constant when the frequency is large enough,
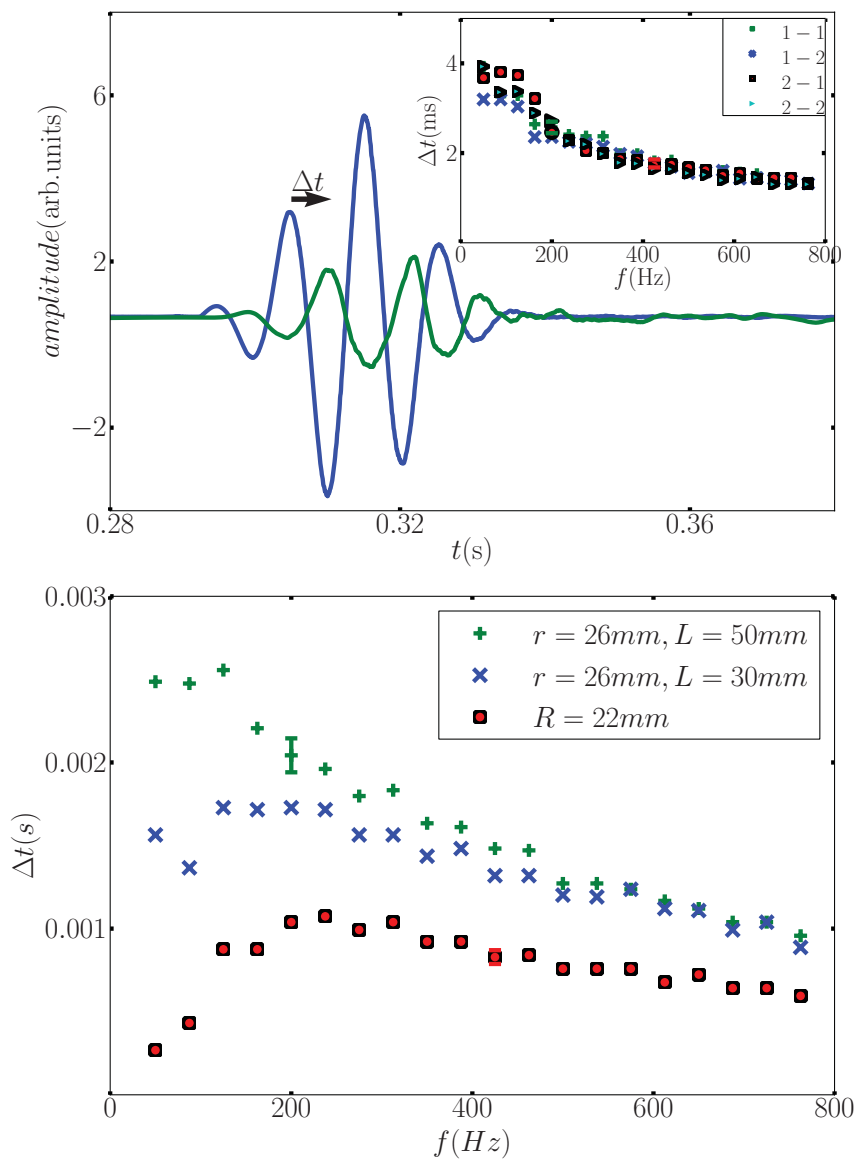

FIG. 2. (Color online) (a) Typical signals: Input at the lower accelerometer and output at the upper accelerometer. The flight time $\Delta t$ is defined as the delay of the first acceleration extremum from input to output. Inset: Flight time vs forcing frequency for two cylindrical samples produced independently but with the same geometrical parameters: from square foils of side length $330 \mathrm{~mm}$ to final dimensions $26 \mathrm{~mm}$ and $L=30 \mathrm{~mm}$. Two repetitions are shown for each sample: + and $\times$ for the first; $\circ$ and $\triangleright$ for the second sample. (b) Flight time $\Delta t$ vs forcing frequency $f$ for three crumpled samples with different geometrical parameters (see the inset). All sheets used were squares of side $330 \mathrm{~mm}$. Typical error bars are shown.

consistently with a quadratic dispersion relation there. We take the corresponding plateau as the actual value of $l^{*}$ for bending waves, as compiled in Fig. 4. Surprisingly, we find $l^{*}$ to be smaller than the distance between the sensors. This suggest that two types of propagation occur: free bending waves along some parts of the path and much faster waves along other parts of the path. The increase of $l^{*}$ at small frequencies might be ascribed to the entrainment of air and to a wavelength larger than the distance between the sensors.

In order to rationalize the possibility of fast waves, we consider the structure of the crumpled ball, i.e., the polyhedrallike shape with facets of millimetric size. Indeed, we observed that the effective length decreased when we remodeled a crumpled ball so as to create smaller facets (but keeping the distance between sensors constant). A first rationale is provided by the propagation of bending waves on a nonflat sheet when wavelengths are larger than the characteristic length of the sheet shape. This was investigated in periodic 

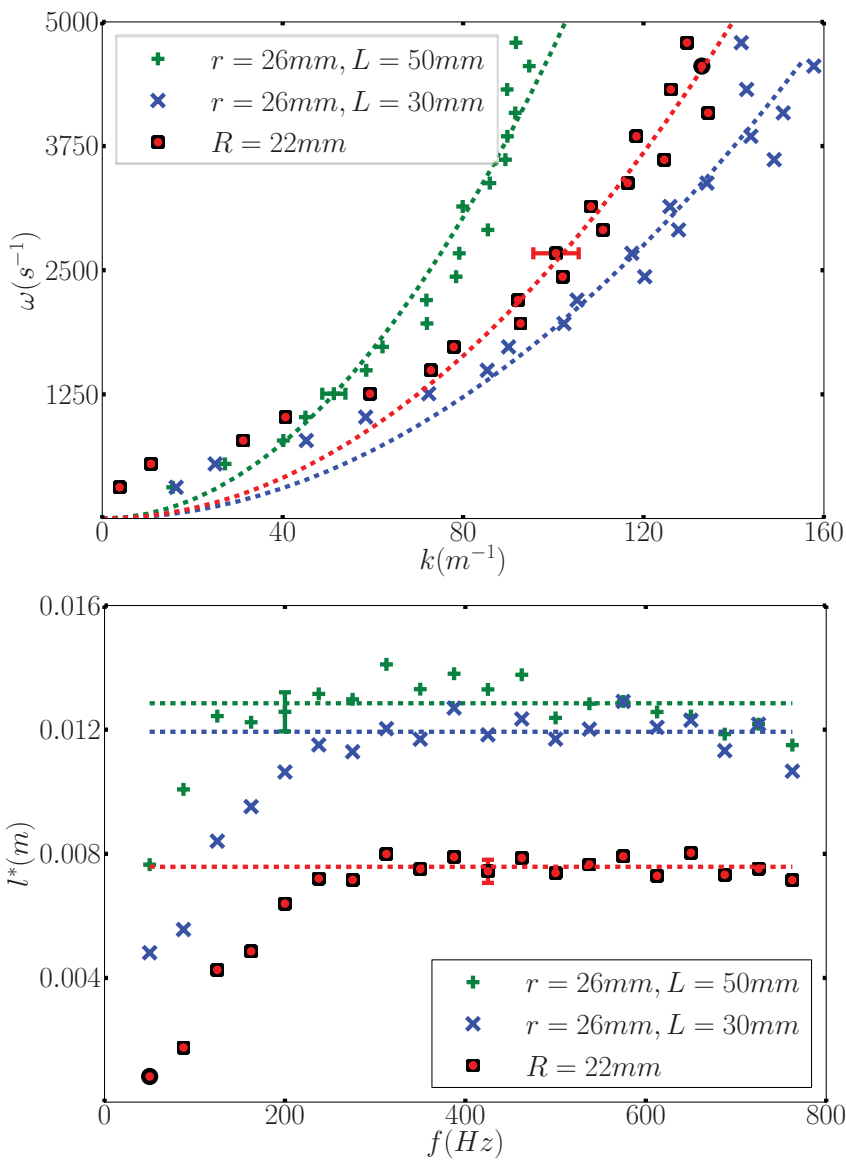

FIG. 3. (Color online) (a) Examples of raw dispersion relations. Angular frequency $\omega=2 \pi f$ vs wave number $k$ and quadratic fits. The data comes from the same crumpled samples as in Fig. 2(b). (b) Corresponding effective path length $l^{*}$ vs frequency $f$. Typical error bars are shown.

geometries where all folds are parallel, in the context of corrugated plates (e.g., Ref. [21] and references therein). The main result was that the effective bending stiffness in the direction parallel to folds is much larger than the effective bending stiffness in the direction normal to the folds. Transposing to our geometry where the characteristic length is given by facet size $W$, the ratio between these two stiffnesses is of the order of $(W / h)^{2}, h$ being the thickness of the sheet. Consequently, the dispersion relation in the direction parallel to the folds is obtained from Eq. (1) by replacing $h=20 \mu \mathrm{m}$ with a length of the order of $W \sim 2 \mathrm{~mm}$, making bending waves in the direction of folds approximately 100 times faster than free bending waves. This factor of 100 seems too high to account for the prefactors of the dispersion relations of Fig. 3(a), which are approximately ten times larger than for free bending waves. However, a combination of propagation parallel and perpendicular to folds could account for these dispersion relations. Overall this crude argument indicates that the sheet geometry may make propagation fast along one direction (due to a much higher effective bending stiffness) and slow along the other direction (with a velocity comparable to that of free bending waves).

A second rationale stems from air compressibility. The stiffness of the spring equivalent to an air cavity of size $\ell$

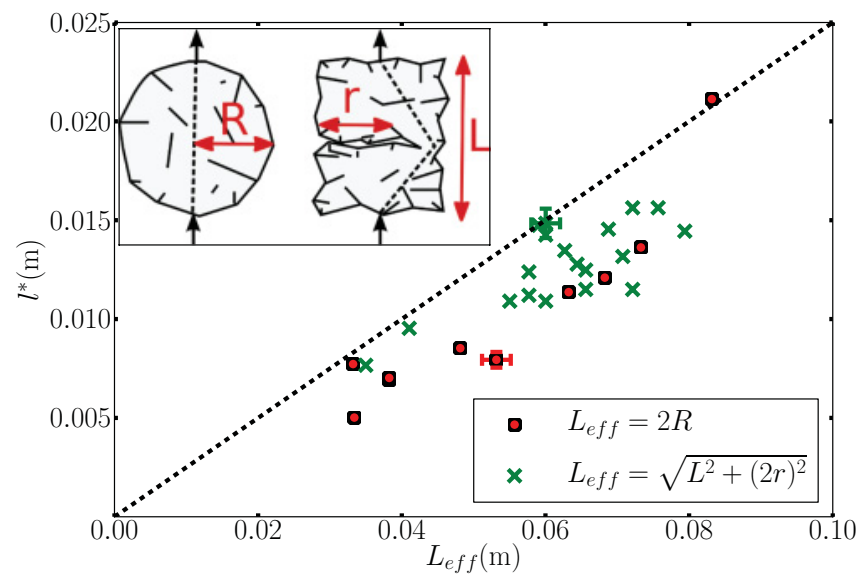

FIG. 4. (Color online) Propagation path length. Inset: A schematic of propagation paths (dashed lines). Left-hand side: Straight propagation in a ball. Right-hand side: Configuration of a cylinder. The initial cone has been folded in two, yielding a cylinder with a slit parallel to its faces. A path between the two sensors must go around the slit, covering a distance $\sqrt{L^{2}+(2 r)^{2}}$. Main figure: Path length $l^{*}$ extracted from the plateau [see Fig. 3(b)] for different crumpled samples as a function of the effective distance $L_{\text {eff }}$ between the two sensors: the diameter $2 R$ for spherical samples $(\bullet)$, and the distance around the slit $\sqrt{L^{2}+(2 r)^{2}}$ for cylindrical samples $(\times)$; the samples were obtained from square sheets of side in the range 200-330 mm.

and pressure $P$ is of the order of $P \ell$, while the stiffness of a facet of size $\ell$ is $E h^{3} / \ell^{2}, E$ being the elastic modulus of aluminum. The stiffness of the cavity becomes dominant when its size $\ell$ is larger than $\ell_{c}=(E / P)^{1 / 3} h \sim 1 \mathrm{~mm}$, which is smaller than facet sizes in experiments. This would imply that all propagation occurs through air compression, leading to nondispersive waves in a material having the compressibility of air and the average density of the ball, at velocities of the order $100 \mathrm{~m} / \mathrm{s}$, much larger than in our observations. We thus favor the first rationale, although we cannot exclude a more subtle situation with a mix of propagation through air cavities and bending waves. In any case, we expect a combination of free bending waves and faster waves.

In Fig. 4, the path length for spherical samples appears to be $\sim 1 / 4$ of the distance between the sensors. In the framework discussed above, this fraction corresponds to the probability of having a slow direction pointing toward the output sensor at a given facet. We found that cylindrical samples have a different dependence of $l^{*}$ on $L$ than spherical samples, which can be accounted for as follows. The effective distance between the sensors $L_{\text {eff }}$ is just the diameter for the spherical sample balls but it is more complex for cylinders. Recall that cylindrical samples were obtained from the folding of a cone, so that they generally have a slit in the middle (the inset of Fig. 4), as can be checked by unfolding the configuration. This slit might prevent direct propagation between the sensors, therefore, we also tried the shortest path around the slit, yielding a length $L_{\text {eff }}=\sqrt{L^{2}+(2 r)^{2}}$, as schematized in the figure. The result is shown in Fig. 4 and collapses with the data for spherical samples, suggesting that the large-scale structure of the sample is important for path selection. We finally note that $l^{*}$ is much more correlated to the effective distance between the 
sensors than to the packing fraction, which changes by a factor 20 across all samples, indicating that there are enough facets to ensure the continuity of the path between the two sensors.

To conclude, we investigated the propagation of waves in a crumpled ball; the waves appear to reflect both the smalland the large-scale structure of the ball. We found that the raw dispersion relation varies with the samples, and that the waves travel faster than free bending waves. This seems in contrast with the numerical work in Ref. [16], showing trapping of waves in the facets of a tetrahedron, but this discrepancy can be easily explained knowing that the wavelengths were smaller than the size of the tetrahedron, while we forced wavelengths much larger than the size of the facets in the crumpled ball. We interpreted our results in terms of fast and slow directions, respectively parallel and perpendicular to folds. This interpretation could be used to identify unique geometrical properties of crumpled balls.
[1] T. A. Witten, Rev. Mod. Phys. 79, 643 (2007).

[2] F. Plouraboué and S. Roux, Physica A 227, 173 (1996).

[3] D. L. Blair and A. Kudrolli, Phys. Rev. Lett. 94, 166107 (2005).

[4] C. A. Andresen, A. Hansen, and J. Schmittbuhl, Phys. Rev. E 76, 026108 (2007).

[5] M. A. F. Gomes, C. C. Donato, S. L. Campello, R. E. de Souza, and R. Cassia-Moura, J. Phys. D 40, 3665 (2007).

[6] A. S. Balankin, D. Morales Matarnoros, E. Pineda Leon, A. Horta Rangel, M. A. Martinez Cruz, and D. S. Ochoa, Physica A 388, 1780 (2009).

[7] T. Tallinen, J. Åström, and J. Timonen, Nat. Mater. 8, 25 (2008).

[8] G. A. Vliegenthart and G. Gompper, Nat. Mater. 5, 216 (2006).

[9] E. Sultan and A. Boudaoud, Phys. Rev. Lett. 96, 136103 (2006).

[10] K. Matan, R. B. Williams, T. A. Witten, and S. R. Nagel, Phys. Rev. Lett. 88, 076101 (2002).

[11] Y. C. Lin, Y. L. Wang, Y. Liu, and T. M. Hong, Phys. Rev. Lett. 101, 125504 (2008).

[12] P. A. Houle and J. P. Sethna, Phys. Rev. E 54, 278 (1996).
[13] E. M. Kramer and A. E. Lobkovsky, Phys. Rev. E 53, 1465 (1996).

[14] R. S. Mendes, L. C. Malacarne, R. P. B. Santos, H. V. Ribeiro, and S. Picoli, Europhys. Lett. 92, 29001 (2010).

[15] A. Lagendijk, B. A. van Tiggelen, and D. S. Wiersma, Phys. Today 62(8), 24 (2009).

[16] A. Gopinathan, T. A. Witten, and S. C. Venkataramani, Phys. Rev. E 65, 036613 (2002).

[17] A. Boudaoud, E. Hamm, and F. Melo, Phys. Rev. Lett. 99, 254301 (2007).

[18] L. Boué, M. Adda-Bedia, A. Boudaoud, D. Cassani, Y. Couder, A. Eddi, and M. Trejo, Phys. Rev. Lett. 97, 166104 (2006).

[19] S. Deboeuf, M. Adda-Bedia, and A. Boudaoud, Europhys. Lett. 85, 24002 (2009).

[20] L. Landau and E. Lifshitz, Theory of Elasticity, 3rd ed. (Pergamon, New York, 1986).

[21] D. Wennberg, P. Wennhage, and S. Stichel, ISRN Mech. Eng. (2011) 979532. 